\title{
The Firing of Hippocampal Place Cells Predicts the Future Position of Freely Moving Rats
}

\author{
Robert U. Muller ${ }^{1}$ and John L. Kubie ${ }^{2}$ \\ 'Department of Physiology and 'Department of Cell Biology and Anatomy, SUNY Health Sciences Center at Brooklyn, \\ Brooklyn, New York 11203
}

\begin{abstract}
Direct observation and automatic, video-based methods reveal that a large fraction of hippocampal pyramidal neurons recorded from freely moving rats behave as "place cells"; the firing of each place cell occurs almost exclusively when the rat is in a restricted part of its current environment. In earlier work, 2-dimensional firing distributions for place cells over the apparatus area were made under the assumption that the correct location for each spike was the animal's position at the instant that the spike was fired. Spatial firing distributions generated in this way often have a very simple structure, in which the single region of intense activity has a just one maximum, and where the rate decreases monotonically in all directions away from the maximum. We will refer to patterns of this sort as "ideal."

We describe how the spatial firing pattern is altered by assigning spikes to positions earlier or later than the instant at which they were fired. Spatial firing distributions were generated for a range of constant displacements of the spike time-series against the position time series. Three quantitative measures were used to estimate the extent to which the spatial firing pattern at different "spike/position shifts" approximated the ideal pattern. The 3 measures are in agreement that spikes must precede the animal's position by about $120 \mathrm{msec}$ for the spatial firing pattern to be closest to the ideal. These results suggest that hippocampal unit activity predicts the animal's future location on a short time scale.
\end{abstract}

Place cells, a large subset of hippocampal pyramidal cells, are characterized by their tendency to fire rapidly only when a freely moving rat is in a certain portion of the space to which the animal has access (O'Keefe and Dostrovsky, 1971; O'Keefe, 1979). Direct observation indicates that the firing is mainly a function of the animal's position and not of the animal's moment-to-moment activity. That the firing may be location-specific rather than behavior-specific was demonstrated by showing that regions of restricted firing occur even when the animal's behavior is quite homogeneous in time and space (Muller et al., 1987).

\footnotetext{
Received Sept. 6, 1988; revised May 9, 1989; accepted May 19, 1989.

We thank Drs. Elizabeth Bostock and Pat Sharp for providing some of the data used in this paper. We also thank Dr. James B. Ranck, Jr., for reading the manuscript of this paper and for his assistance in outlining the most direct known paths between the hippocampus and motoric regions of the rat brain. The work in this paper was supported in part by NIH grant R01-NS20686.

Correspondence should be addressed to Dr. Robert Muller, Department of Physiology, Box 31, SUNY Health Sciences Center at Brooklyn, 450 Clarkson Ave., Brooklyn, NY 11203.

Copyright (c) 1989 Society for Neuroscience $0270-6474 / 89 / 124101-10 \$ 02.00 / 0$
}

The degree of location-specificity of place cell firing is visible in Figure $1 A$, which is a color-coded map of the time-averaged firing rate of a single cell recorded as a rat moved around for $16 \mathrm{~min}$ in a $76-\mathrm{cm}$-diameter, $51-\mathrm{cm}$-high cylinder. In Figure $1 A$, the region of high firing rate-the "firing field"-is the nonyellow region at the cylinder edge near 5:00 o'clock. The specificity may be appreciated from the fact that the firing rate averaged $0.44 \mathrm{spikes} / \mathrm{sec}$ outside the field and $8.8 \mathrm{spikes} / \mathrm{sec}$ in the field.

In previous work on place cells, it was tacitly assumed that spikes are properly bound to the animal's position at the instant that the spikes occur. It is possible, however, that spikes are more correctly associated with positions of the animal earlier or later than the moment at which they are fired, as might be expected from the existence of propagation and synaptic delays between peripheral and central events. The main purpose of this paper is to describe how spatial firing distributions change in appearance when a temporal lead or lag between spikes and position is introduced. The temporal displacement will be referred to as the "spike/position shift." It is our contention that the "proper" spike/position shift is that shift at which the spatial firing pattern for a cell is least noisy.

The method of time-shifting spikes against positions is straightforward. Data are collected (at $60 \mathrm{~Hz}$ ) as a sequence of locations and spike counts; each sample is composed of a position measurement and an associated number of action potentials fired in the $1 / 60$ th sec since the previous position measurement. The data may be thought of as 2 independent time series which can be displaced relative to each other by sliding the entire spike series against the position series by a certain time interval. If the spikes are made to lead position, the shift is taken as positive; if the spikes lag position, the shift is negative. Since each spikc/position shift yields a different spatial firing distribution, the distributions that result from a range of delays may be compared to find the "cleanest" one.

It will be shown that with large spike/position shifts of either sign, spatial firing patterns are strongly degraded relative to patterns with zero shift; Figure $1 B$ shows how the spatial firing pattern in Figure $1 A$ is smeared by a shift of $-1.0 \mathrm{sec}$, and Figure $1 C$ shows the effects of a shift of $+1.0 \mathrm{sec}$. More precise methods than inspection of rate maps are required, however, to distinguish between spatial firing patterns generated by shifts that differ by only a few sample intervals. Three quantitative measures of firing pattern clarity are used to search for the optimal spike/position shift. The use of 3 methods reduces the likelihood that any interesting results are due to a hidden defect of a single method.

The 3 measures of firing pattern quality are based on the idea 


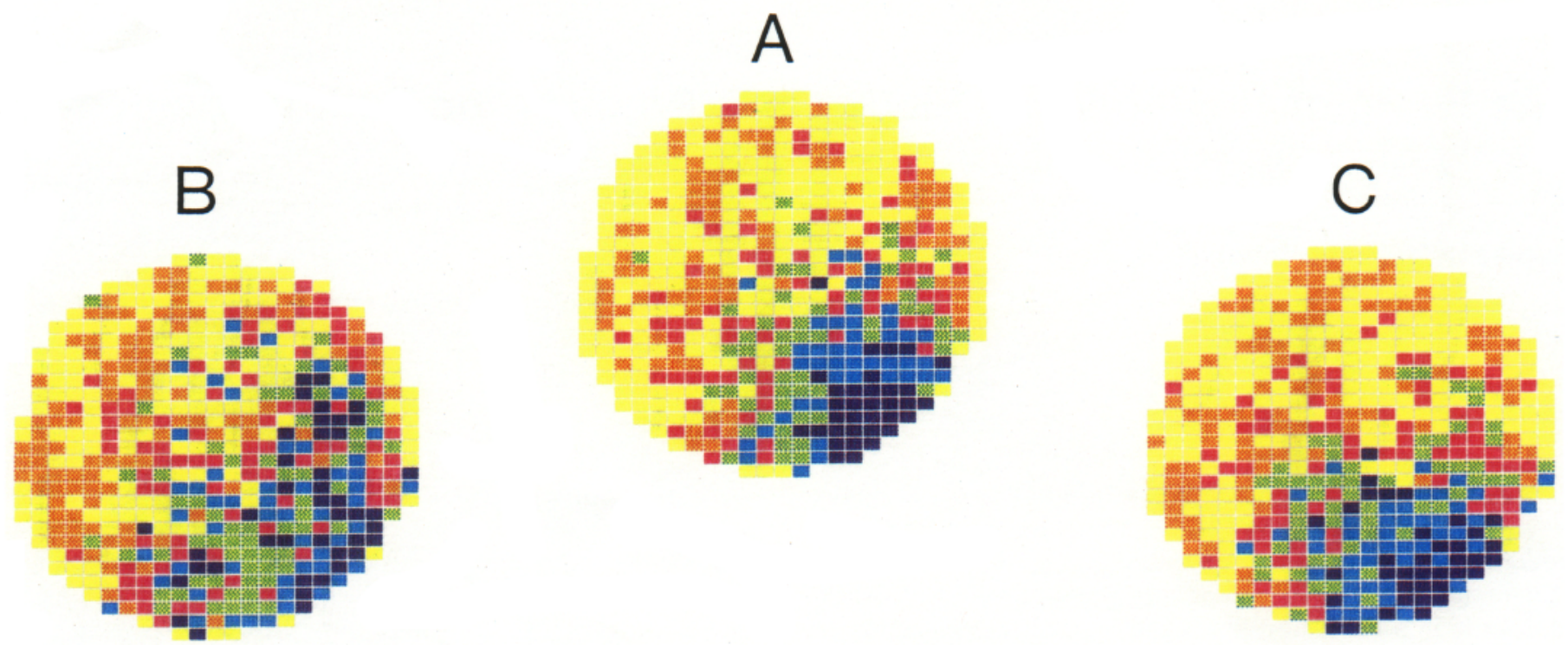

Figure 1. Color-coded representations of the spatial firing patterns generated by a place cell after shifts of $0.0,-1.0$, and +1.0 sec. $A$, Rate map at $0.0 \mathrm{sec}$ delay approximates the idealized spatial firing pattern described in the introduction. By this standard, the firing patterns at -1.0 sec $(B)$ and $+1.0 \mathrm{sec}(C)$ are noticeably degraded, although the amount of degradation is less for the +1.0 -sec shift. Rat 53 , session 6 . The median firing rates for color-coded firing rate categories were: yellow, 0.0; orange, 0.97; red, 3.00; green, 6.53; blue, 15.0; purple, 26.7.

that there is an ideal spatial firing pattern for place cells (Muller et al., 1987). The ideal pattern is made up of just one firing field, within which the firing rate decreases monotonically in all directions from a single maximum. In the ideal case, the firing rate outside the field is exactly zero. The firing rate map of Figure $1 \mathrm{~A}$ illustrates a firing pattern that closely approximates our notion of the ideal.

In the results, we show that the 3 measures of spatial firing pattern quality behave as expected, in that each reveals degradation with large spike/position shifts of either sign. In addition, the methods agree that firing pattern quality is optimized at positive values of the spike/position shift, averaged across the cell sample. The implications of the fact that the cleanest patterns are produced with positive shifts are considered in the Discussion, along with possible applications of the spike shift method to other areas of neurophysiology.

\section{Materials and Methods}

The procedures used to record hippocampal neurons in freely moving animals are presented in detail in Kubie (1984) and Muller et al. (1987). Accordingly, only a summary of the general methods is given here. Most of this section is devoted to describing the cell sample, the schemes developed to measure the clarity of spatial firing patterns, and consideration of persistence in the video camera as a source of error in estimating optimal spike/position shifts.

General methods. We record the firing of place cells in a wooden cylinder $76 \mathrm{~cm}$ in diameter and $51 \mathrm{~cm}$ high. It is uniformly gray except for a white cardboard "polarizing" stimulus that runs from top to bottom and that occupies $100^{\circ}$ of arc, centered at 3:00 o'clock in the overhead view from a TV camera. The apparatus is placed on a sheet of gray photographic back-drop paper that is changed before each recording session. During sessions, the cylinder is centered in a set of dark curtains that visually isolate the animal from the rest of the laboratory room. Many of the cells were also recorded when the animal was in one or more variants of the cylinder, but only "small" cylinder sessions are considered here.

A rat is encouraged to visit all parts of the cylinder during a 16-min experimental session by teaching it to chase small food pellets that are scattered into the cylinder by throwing them over the curtain at a rate of 2 or $3 / \mathrm{min}$. Thus, the rat is not required to treat any part of the apparatus as special, and its behavior is as homogeneous in space and time as we have been able to arrange. To ensure a constant behavioral state from rat to rat and during each session, the animals are trained in this task for about 2 weeks before any data are taken.

During a session, 2 aspects of the animal's state are measured. A television camera and a home-made interface are used to digitize the position of 2 small incandescent lights placed side-by-side on the animal's headstage. The position of the headlights is found in a $64 \times 64$ grid of rectangular subelements (pixels) of the video field, each of which is $3.4(X) \times 2.8(Y) \mathrm{cm}$ in size. The small cylinder occupies $12 \%$ of the video field and is about 510 pixels in area. The position is detected during each TV field, at a frequency of $60 \mathrm{~Hz}$.

In addition to tracking the animal's position, the action potential traffic from a single place cell is recorded. Place cells are hippocampal pyramidal cells that are found in either regio superior or inferior. As in previous work from this and other laboratories, recordings were confined to the dorsal third of the hippocampus. The moveable electrode array is aimed to cross the pyramidal cell layer of regio superior (CA1) at coordinates: $4.0 \mathrm{AP}, 7.2$ above ear bar zero, and $3.0 \mathrm{~mm}$ lateral, according to (Atlas ref). The array is mounted at an $8^{\circ}$ angle so that the tip moves lateral to medial in a fixed coronal plane and passes through the pyramidal cell layer of regio inferior (CA3) at coordinates: $4.0 \mathrm{AP}$, 6.2 above ear bar zero and $2.8 \mathrm{~mm}$ lateral. Electrode and surgical specifics are given in Kubie (1984) and Muller et al. (1987).

Signals from cells with distinctive waveforms are amplified, bandpass-filtered between 300 and $10,000 \mathrm{~Hz}$, and sent to a series of 3 timeand-amplitude window discriminators. Waveforms that trigger all 3 discriminators in succession are called action potentials. The acceptance pulse of the final discriminator increments a 4 bit counter that is set to zero at the start of each TV field. In other words, only the number of action potentials fired during each field is counted. The number of spikes and the location information are sent to a small computer each 16.7 msec. During a 16-min recording session, 57,600 location and spike count samples are gathered. Data analysis is done off-line on a Masscomp 5500 minicomputer.

For analysis, the amount of time the animal spends in each pixel and the number of spikes fired in each pixel are accumulated, respectively, into a $64 \times 64$-"time-in-location" array and a $64 \times 64-$ "spikes-inlocation" array. A time-averaged firing rate array is made by dividing the spike array by the time array element by element. A color-coded rate map is printed when it is desired to visualize the firing rate array. Each element in the rate array is put into one of 7 categories. White signifies an indeterminate rate for pixels not entered by the rat. Since nearly every pixel in the cylinder is visited during a 16-min session, the cylinder's area appears as a colored circle on a white background. Unvisited pixels in the apparatus are also coded white. Color encodes increasing firing rate in the order: yellow, orange, red, green, blue, purple. Yellow represents regions of exactly zero firing rate. The breakpoints 
between the other categories are not fixed from session to session. Instead, the breakpoints are set so that the number of pixels assigned to a category is equal to 0.8 times the number of pixels in the next lower firing rate category. This rank-scaling ensures that all 5 nonyellow colors will be used to cover the full firing rate range, independent of the absolute firing rate. The 0.8 ratio for the number of pixels printed in adjacent color categories means there will be about 0.4 times as many purple as orange pixels, making it easier to pick out regions of relatively high rate.

Cell selection. The fraction of adequately discriminated complexspike cells that we call place cells is around $50 \%$. The sample of 90 cells was selected from a total of about 350 by rejecting cells that (1) did not have fields in the cylinder; (2) had 2 clear-cut fields; (3) had "noisy" or weak firing fields. These judgments were made from inspection of firing rate maps together with numerical firing rate values for the color categories. We have not investigated the effects of shifts on cells whose spatial firing patterns did not meet these criteria.

Spike/position shift. The spike/position shift treats the sequences of spikes and positions from a recording session as separable time series which may be displaced by a certain amount relative to each other. In practice, we choose to imagine that the position series stays fixed and the spike series is moved forward or back in time. The shift is called positive if spikes are moved forward in time and negative if spikes are moved backwards. Once the shift is made, time-in-location, spike and firing rate arrays are, made just as for the original (zero shift) data. A firing rate array is constructed for each spike/position shift in the range from -1.0 to $+1.0 \mathrm{sec}$, in $1 / 60$ th-sec increments. The rate arrays are then compared to see which contains the clearest firing pattern. Firing pattern quality is measured in 3 ways.

Firing area as an estimate of firing pattern quality. The first method for optimizing spatial firing clarity is to find the spike/position shift that minimizes the number of pixels in which the firing rate was greater than zero. We argue that minimizing firing area optimizes pattern quality in the sense that spatial firing specificity is then highest. If the minimum firing area occurred at 2 or more shifts, the value of the minimum was taken as the arithmetic mean of the individual shifts.

Patchiness as an estimate of firing pattern quality. The second method of optimizing spatial firing patterns seeks the shift associated with the smoothest large-scale contours by minimizing the number of iso-firing rate regions or "patches." A patch is defined as a continuous set of pixels ( $\geq 1$ pixel) whose members all have firing rates in the same range. For inclusion in a patch, a candidate must share at least an edge with a pixel known to be a member of the patch; a corner is not enough. The firing rate range for each class is generated by the autoscaling algorithm stated above, so that patches may be counted directly from a colored map. For the zero-delay map of Figure $1 A$, there are 5 purple patches, 11 blue patches, 29 green patches, etc. Since firing rate is divided into ranges, patches could be counted in several ways. For example, it is possible to count the number of purple patches, the number of purple + blue patches, etc. We find, however, that the basic conclusion is unchanged regardless of which sum is chosen. The measure used is to count the patches for the highest (purple) rate category, since it is easiest to check visually from rate maps. Because of the extremely tight binding of firing to position, almost all patches in the highest rate category are found in the main firing field with shifts near zero.

Spatial coherence as an estimate of firing pattern quality. The third estimate of orderliness of the spatial firing distribution is a first-order spatial autocorrelation that will be called "coherence." Coherence is the $z$-transform of the correlation between a list of firing rates in each pixel and a corresponding list of firing rates averaged over the 8 nearestneighbors of each pixel. Coherence measures the extent to which the fring rate in a pixel is predicted by the rates in its neighbors, and therefore estimates the local orderliness of the spatial firing pattern. Again, because of the tight binding of firing to position, the coherence is greatly determined by the firing within the main field at shifts near zero.

Effects of image persistence in the TV camera. The Nuvacon TV camera (Panasonic model WV-1350A) used to track the rat's headlights may introduce a temporal error in the animal's recorded position due to image persistence. If it is severe enough, persistence can be seen as a tail that follows a bright object moving on the TV screen. Since the headlight position is taken as the first pixel in which the light intensity is above the detection threshold, it is possible for the animal's recorded position to lag its true position. This can happen if the animal moves down or to the right in the TV field, since the field is scanned from upper left to lower right. If persistence were of major importance, it would produce the equivalent of a positive spike/position shift in our
Table 1. Amount of shift necessary to optimize 3 field quality measures: tube and solid-state cameras

\begin{tabular}{|c|c|c|c|c|c|}
\hline Measure & $\begin{array}{l}\text { No. of } \\
\text { cells }\end{array}$ & $\begin{array}{l}\text { Mean } \\
\text { shift }^{a}\end{array}$ & SD & $t^{b}$ & $P(t)$ \\
\hline \multicolumn{6}{|l|}{ Tube camera } \\
\hline Firing area & 70 & 104.5 & 168.9 & 5.47 & $3.4 \mathrm{E}-7$ \\
\hline Patchiness & 70 & 113.2 & 169.7 & 5.58 & $2.2 \mathrm{E}-7$ \\
\hline Coherence & 70 & 113.5 & 184.7 & 5.15 & $1.2 \mathrm{E}-6$ \\
\hline \multicolumn{6}{|c|}{ Solid-state camera } \\
\hline Firing area & 20 & 126.2 & 234.8 & 2.40 & $1.3 \mathrm{E}-2$ \\
\hline Patchiness & 20 & 137.8 & 170.0 & 3.63 & 8.9E-4 \\
\hline Coherence & 20 & 164.2 & 172.3 & 4.17 & $2.6 \mathrm{E}-4$ \\
\hline
\end{tabular}

${ }^{a}$ Means and SD for optimizing shifts are in msec.

- The $t$ values are calculated from the null hypothesis that the mean optimizing shifts are zero.

analysis; it would be necessary to shift the spike time series forward in time to compensate for the delayed position values. Given that fields are, on the average, optimized at positive values of the shift, it is crucial to measure the magnitude of the persistence effect.

To this end, a motorized, 91.4-cm-long conveyor belt was constructed. Mounted on the belt was a small incandescent bulb of the same type attached to the rat's headstage, a resistor to set the light intensity, and 3 AA batteries to power the light. The belt was driven at speeds up to $132 \mathrm{~cm} / \mathrm{sec}$, well in excess of the running speeds observed for rats in the limited confines of the small cylinder.

Mounted next to the conveyor was a phototransistor at the height of the belt. The phototransistor was set so that the light was detected at the transition into a known pixel as viewed by the TV camera. The phototransistor output was amplified and used to activate a Schmitt trigger and a 1-shot. The pulse from the 1-shot was led to the input of the counter used to accumulate spikes. The persistence was estimated by finding the TV pixel in which the moving light was detected by the phototransistor. With this method, the persistence effect was zero within experimental error when the light intensity and TV camera $f$-stop were the same as those used during experimental sessions, even when the light was moving at $132 \mathrm{~cm} / \mathrm{sec}$. As the light intensity was increased and the iris was opened, the persistence became detectable, but only when much more than the usual amount of light was admitted into the camera.

A second test of persistence was to expose the Nuvacon camera to the stationary light for several seconds, to saturate the light's image on the photo-sensitive surface. The light paths to the camera and the phototransistor were then simultaneously occluded, and the times at which the camera and the phototransistor stopped detecting were compared. With the ordinary light intensity and $f$-stop the persistence was no more than 2 video fields or about $33 \mathrm{msec}$. This appears to be the outside limit for the effects of persistence and is unrealistically high, given that the rats spend most of their time moving.

As a final check of the importance of persistence, we recorded from 20 cells while tracking the rat with a solid-state camera (Sony model DC-39) whose photosensitive element was a charge-coupled array. Such a camera is expected to show no image persistence from scan to scan, and in fact, none was detected with the occlusion test described above. As documented in Table 1, the field-quality measures yielded similar results with the tube and solid-state cameras; the mean optimizing shifts were, in fact, somewhat larger for the solid-state camera. Since $t$ tests for differences of the mean shifts obtained with the 2 cameras did not approach the 5\% for any of the field quality measures, we combined the samples so that the overall number of cells is 90 .

\section{Results}

Qualitative description of the spike/position shift effect

For every cell, the relationship between spatial firing pattern quality and the spike/position shift has the form expected from elementary considerations; the pattern is optimal with shifts near zero and degrades with large shifts either sign. The variations of firing area, patchiness, and coherence with shifts over the range -1.0 to $+1.0 \mathrm{sec}$ are shown for 3 cells in Figure 2 . 
FIRING AREA
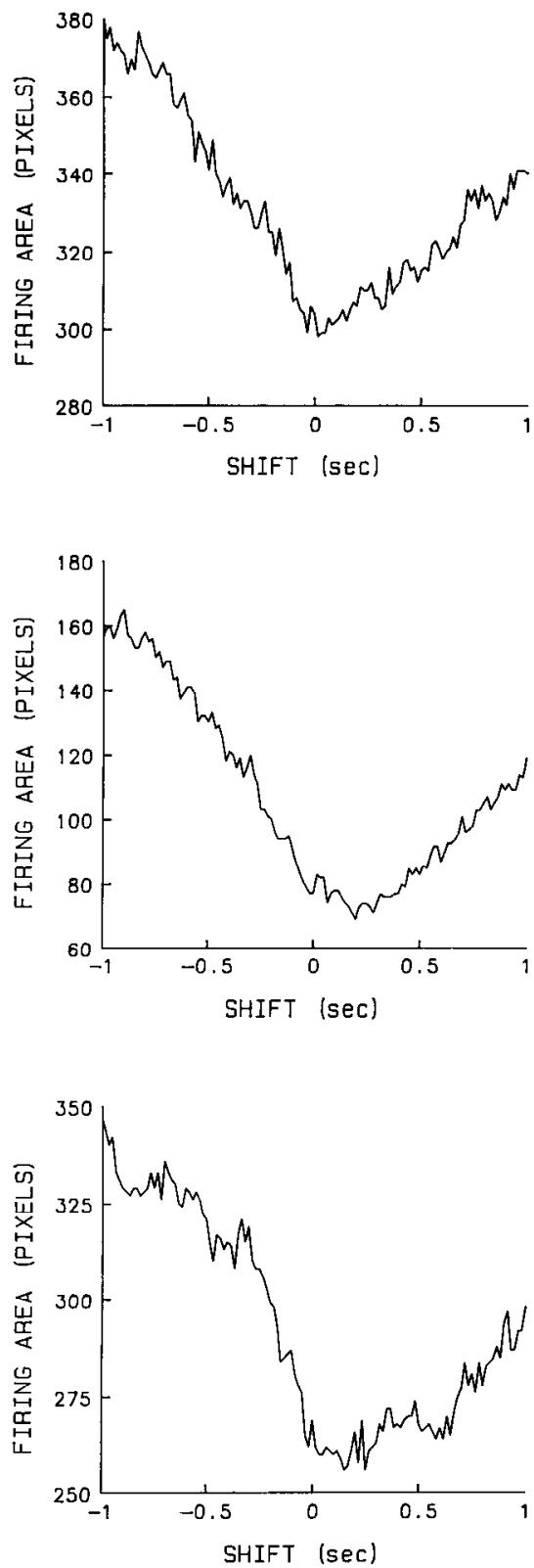

PATCHINESS
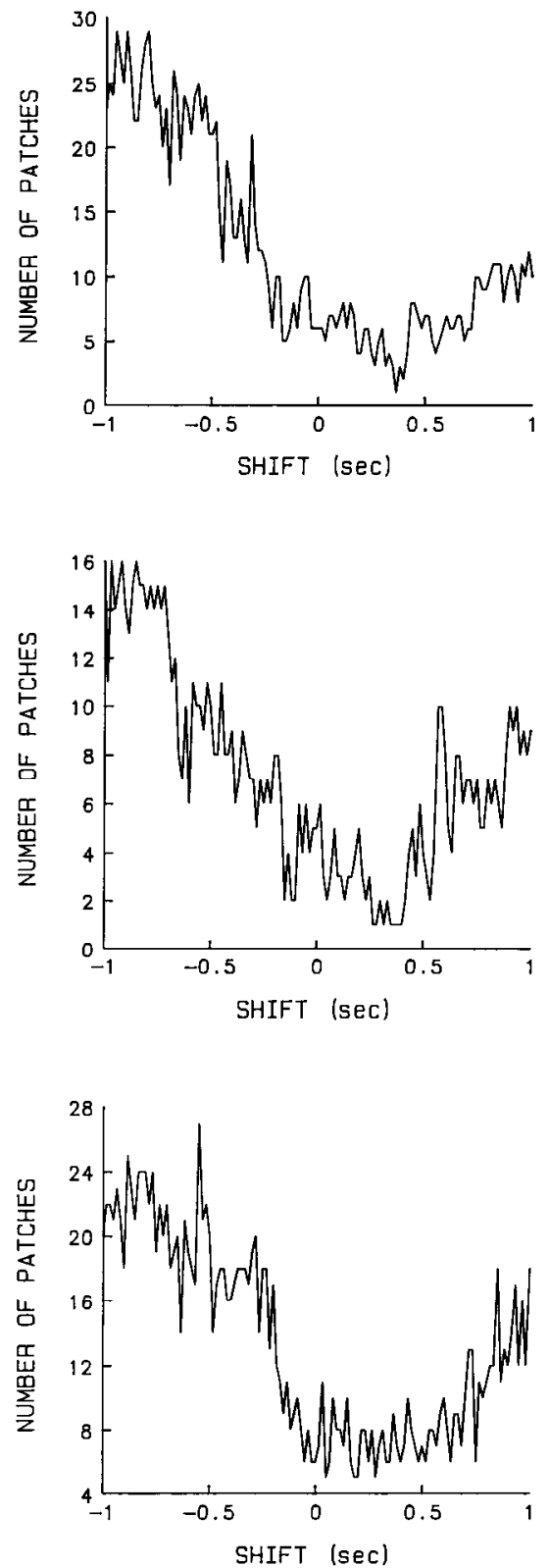

COHERENCE
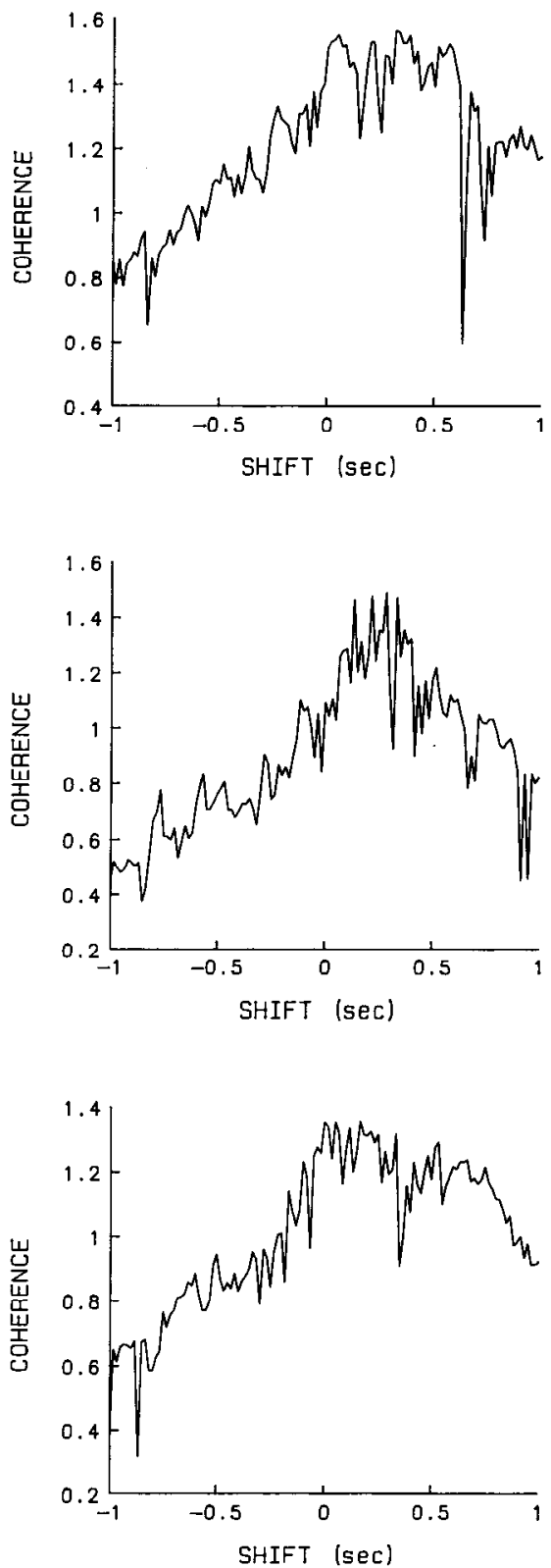

Figure 2. Field quality plotted against spike/position shift for 3 place cells. Each row shows the 3 field quality measures for a single cell over the range -1.0 to $+1.0 \mathrm{sec}$ in $1 / 60$ th-sec steps. The left column shows firing area as a function of shift. Typically, the shift relationship with firing area was smoother than for patchiness (middle column) and coherence (right column). The cells were selected because each field quality measure reached an optimum at a positive shift, and because each measure degrades more rapidly with negative shifts. The selected cells are the same ones whose spatial firing patterns are illustrated in Figure 3.

From these graphs, 2 additional characteristics of the dependence of pattern quality on the shift can be seen. First, for each cell, quality is optimized at a positive shift, for each measure. Second, it appears that the rate of degradation of the pattern is slower for shifts positive to the optima than for shifts negative to the optima.

From the reduced data in Figure 2 alone, it is difficult to gain a sense of the relative orderliness of the spatial firing patterns

Figure 3. Firing rate maps for 3 (rows) place cells at 5 shifts (columns). Column 1, -1.0 sec; column 2, at the negative shift equal in magnitude to the shift at the positive optimum; column 3,0.0 sec; column 4, at the optimum according to minimal patchiness for purple-coded pixels; column $5,+1.0 \mathrm{sec}$. It is our contention that the firing patterns in column 4 are noticeably better than at other shifts for each cell. From the top, the maps in column 4 are for shifts of $0.367,0.350$, and $0.183 \mathrm{sec}$. The shift for the second and third cells were for the integer number of samples that were closest to the mean optimizing shifts, 0.338 and $0.179 \mathrm{sec}$, respectively. In a crude fashion, the maps also illustrate the faster degradation of field quality with negative shifts. Top row, rat 43, session 3. Median firing ratcs: $0.0,1.06,3.53,9.37,15.0,20.5$. Middle row, rat 72, session 1 . Median firing rates: $0.0,0.58,1.58,3.40,7.09,11.2$. Bottom row, rat 85, session 2. Median firing rates: $0.0,0.91,3.05,6.22,10.9,16.7$. 

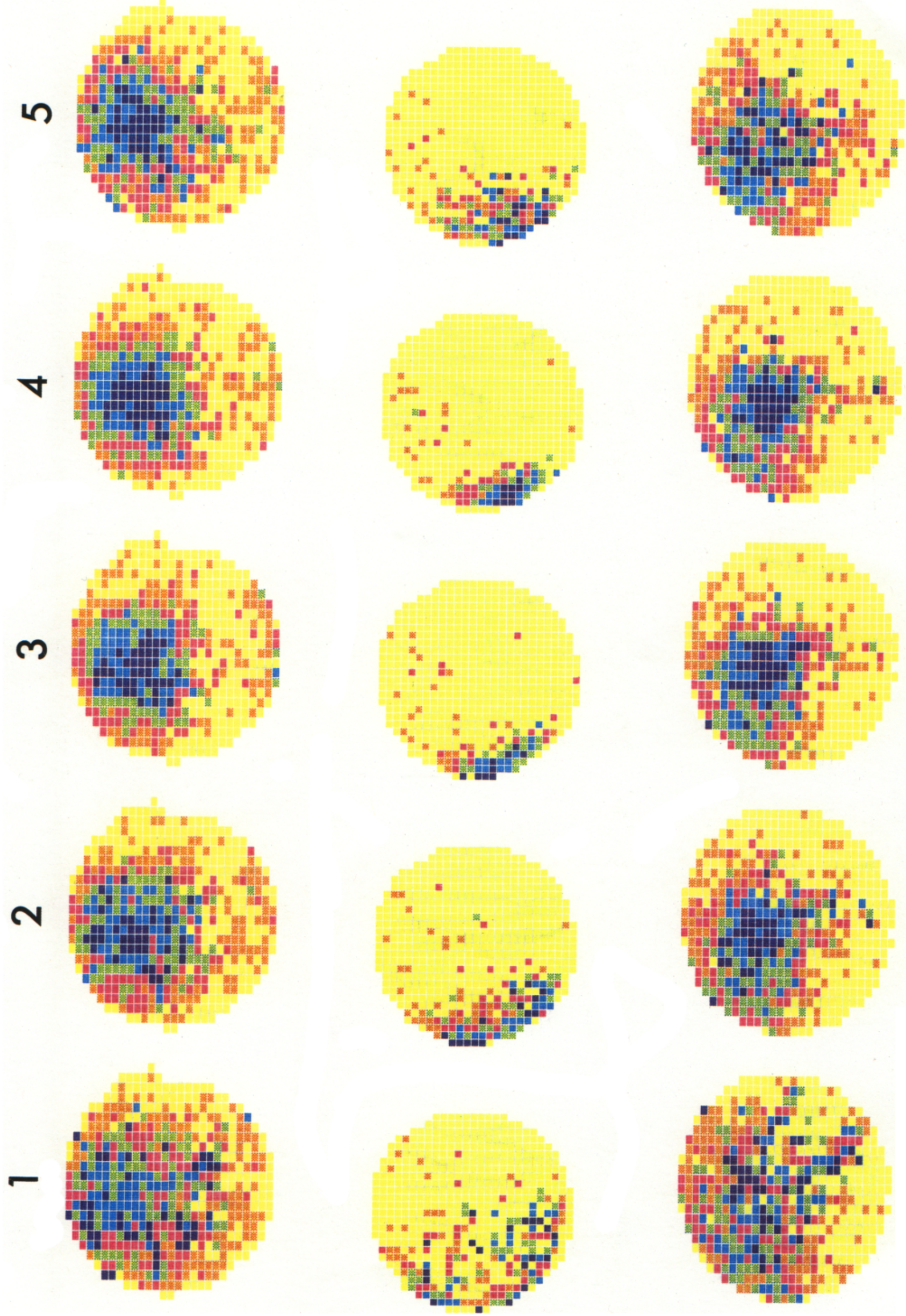


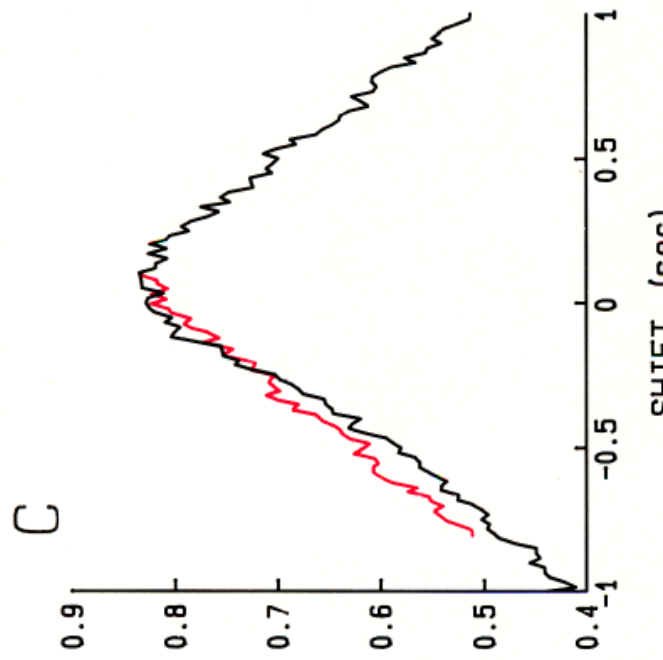

焉

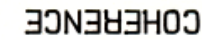
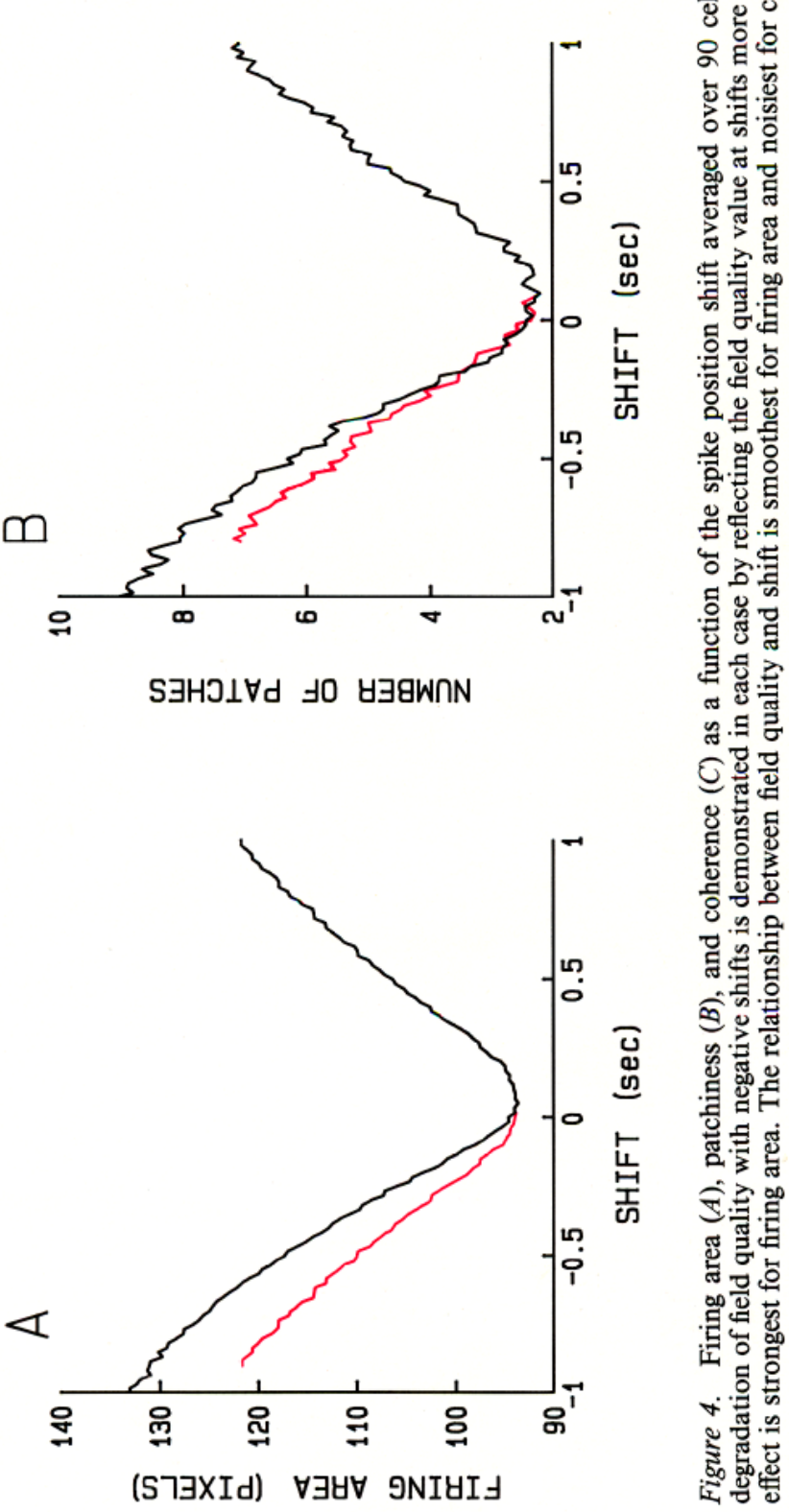
FIAING AREA

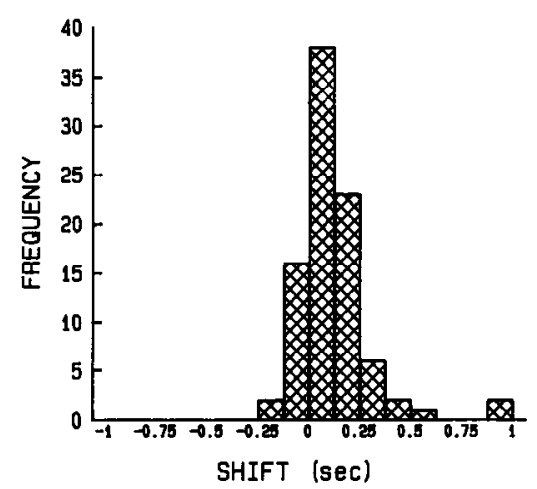

PATCHINESS

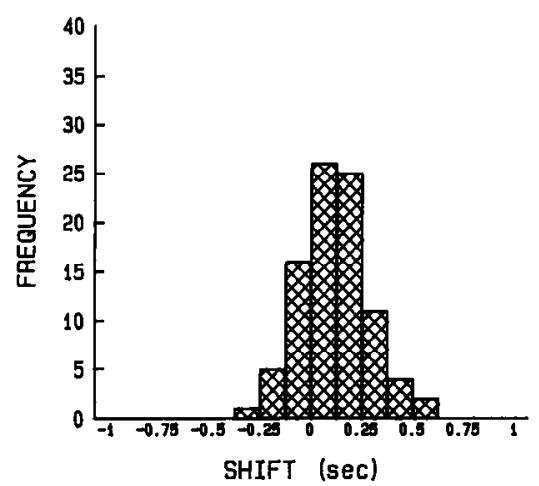

COHERENCE

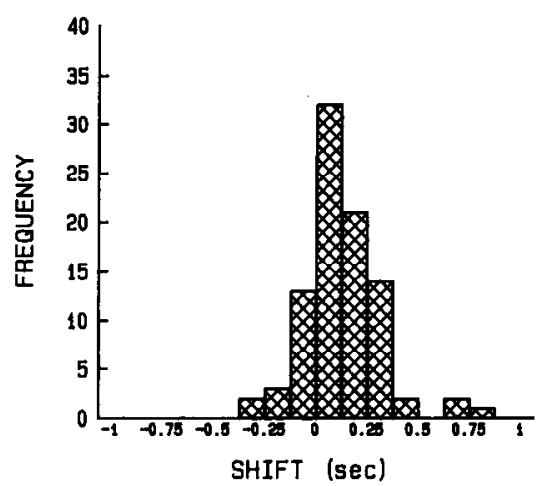

Figure 5. Histograms of shifts that were needed to optimize the field quality of 90 cells, according to each of the measures. The distribution of optimal shifts for each measure approximated a normal distribution with a positive mean very unlikely to have arisen from a population with mean $=0.0$.

at different shifts. The magnitude of the changes in firing pattern quality represented by the formal measures can be judged by inspecting the firing rate maps in Figure 3, which are for the same 3 example cells used in Figure 2. The maps for shifts of -1.0 and $+1.0 \mathrm{sec}$ are noticeably degraded compared with the map for the 0.0 -sec shift. The maps for $-1.0 \mathrm{sec}$ appear to be worse than the corresponding maps for $+1.0 \mathrm{sec}$. Moreover, the maps at the optima (selected for patchiness) are better than at equal negative displacements from a shift of $0.0 \mathrm{sec}$. Finally, the spatial firing patterns in the maps at the optima for patchincss arc visibly improved compared with the firing patterns with the null shift.

The 3 cells used in Figure 3 to illustrate the effects of the spike/position shift were chosen from the 90 cell sample because their spatial firing patterns were optimized with positive shifts, because spatial firing degradation was slower with positive shifts away from the optima, and because the improvement of pattern quality at the optima relative to the null shift was strong enough to be seen in color-coded firing rate maps. For many of the cells, however, the effects of the shift deviated in one way or another from the picture presented so far. For example, it was not uncommon to find that the rate of degradation away from the optima was symmetric or slower with negative shifts according to one or more of the measures. Similarly, the optimum was often nonpositive according to one or more of the measures; 48 cells had positive optima for all 3 measures, 21 for 2 measures, 19 for 1 mcasurc, and 2 cells had no positive optima. According to the binomial distribution, this distribution is extremely unlikely on the hypothesis that negative and positive optima are equally probable.

Although the origins of the varying effects of the spike/position shift are unclear, an averaging procedure shows that the 3 cells described above are reasonable examples for the whole sample. For each of the 90 cells and for each pattern quality measure, the value of the measure at every shift from -1.0 to $+1.0 \mathrm{sec}$ was normalized to the value of the measure at the optimum. For example, the firing area at every shift was divided by the smallest firing area found, so that the normalized values ranged upwards from 1.0. Next, the normalized values were averaged over the cell sample for each shift. Finally, the averaged values were plotted against the shift in Figure 4. To facilitate comparison of the rate of degradation away from the optimum, values of the mcasures at shifts more positive than the optimum were reflected around the optimum.
The averaged relationships between the pattern quality measures and the spike/position shift are similar to the examples shown in Figure 2. Note that the optima are all positive and that the rate of degradation is slower with shifts more positive than the optima. The asymmetry of degradation is seen most clearly with firing area but is in the same direction for patchiness and coherence. The graphs in Figure 4 provide estimates of the amount by which spikes must lead position for spatial firing patterns to be optimal. The estimates of the shift are not very satisfactory, however, for 2 reasons. First, the method constrains the mean shift to be an integer number of sample intervals. Second, and more important, averaging at each shift loses all information about the optimal shift for the individual cells, so that it is impossible to test, for instance, if the size of the average optimal shift is statistically distinguishable from zero. Accordingly, an analysis was made of the distributions of optimal shifts for individual cells.

\section{Statistical description of the spike/position shift effect}

For each cell, the shifts required to optimize the spatial firing pattern were taken as the shift associated with the minimum firing area, minimum patchiness, or maximum coherence. In the event that the optimum for a measure was found at 2 or more shifts, the optimal shift was taken as the arithmetic average of the individual shifts. For instance, if one patch was seen at both +150 and +200 msec for a cell, its optimum for patchiness was taken as $+175 \mathrm{msec}$.

The distributions of optima are summarized in the histograms of Figure 5 and analyzed in Table 2. In Figure 5, it is seen that most of the optimal spatial firing patterns occurred at positive shifts. The probability that the distributions in Figure 5 arose

\begin{tabular}{|c|c|c|c|c|}
\hline Measure & $\begin{array}{l}\text { No. of } \\
\text { cells }\end{array}$ & $\begin{array}{l}\text { Mean } \\
\text { shift }^{a}\end{array}$ & $t^{b}$ & $P(t)$ \\
\hline Firing area & 90 & 113.9 & 5.87 & $\ll 0.001$ \\
\hline Patchiness & 90 & 118.7 & 6.65 & $\ll 0.001$ \\
\hline Coherence & 90 & 124.1 & 6.46 & $\ll 0.001$ \\
\hline
\end{tabular}

\footnotetext{
${ }^{a}$ Mean optimizing shifts are in msec.

${ }^{b}$ The $t$ value is for the null hypothesis that the mean optimizing shift is 0.0 . With 89 degrees of freedom, the probabilities of the $t$ values are on the order of $10^{-8}$.
} 


\begin{tabular}{llll}
\hline \multicolumn{4}{l}{ Table 3. Mean optimizing shifts for CA1 and CA3/4 place cells } \\
\begin{tabular}{llll} 
Measure & No. of cells & Mean shift & SD $^{a}$ \\
\hline CA1 & & & \\
Firing area & 41 & 179.0 & 231.7 \\
Patchiness & 41 & 134.0 & 173.3 \\
Coherence & 41 & 143.1 & 220.0 \\
CA3/4 & & & \\
Firing area & 49 & 59.3 & 107.2 \\
Patchiness & 49 & 105.7 & 158.2 \\
Coherence & 49 & 108.2 & 142.8 \\
\hline
\end{tabular}
\end{tabular}

${ }^{a}$ Means and SD are in $\mathrm{msec}$.

from populations in which positive and nonpositive optima are equally likely approaches zero in each case. In the same vein, the hypothesis that the true optimizing shift is zero is tiny according to the $t$ test (Table 2). Given the near equality of the 3 estimates of the optimal shift, we take their average of +119 msec as the best estimate of the value of the spike/position shift that produces the best spatial firing patterns.

It is also of interest to compare the value of the optimizing shift for individual cells found by the 3 methods. To do this, we calculated the correlation coefficients for the 3 pairs of firing pattern measures. The optima found by patchiness and coherence were fairly highly correlated $(r=0.471)$. By contrast, the correlations between firing area and the other 2 measures were quite low; $r=0.084$ between firing area and patchiness and 0.106 between firing area and coherence. This pattern of correlations arguably validates the conclusions arrived at from the individual measures. In the first place, the fairly high correlation between patchiness and coherence suggests that each indeed reflects an aspect of orderliness in the spatial firing pattern. On the other hand, the low correlations between firing area and the orderliness measures implies that very similar estimates of the mean optimal shift can be generated by measures that are nearly independent of each other.

\section{Statistical analysis of the asymmetry of the shift functions}

The existence of asymmetry in the relationships between the clarity of the spatial firing pattern and shift was tested by comparing the relative clarity at equal shifts before and after the optimum. We averaged each measure for the 30 shifts furthest away from the optimum at positive and negative shifts. The spatial firing pattern was better in the positive direction for 58 / 88 cells according to firing area, for $54 / 90$ cells according to patchiness, and for 53/89 cells according to coherence. From the binomial distribution, the probability of each outcome was $0.003,0.03$, and 0.04 , respectively.

\section{Consistency of the optimal shift within animals and within recording sessions}

Because of the wide variations of the optimal shift for each measure, it is interesting to ask if the optimizing shifts are less likely to vary for cells recorded from individual animals or for cell pairs recorded during a single session. Two or more cells were recorded for 22 of the 44 rats in the sample. If more than 2 cells were recorded from an animal, only the first 2 were used. The correlation coefficients between the mean optimizing shifts for within-animal cell pairs were found to be 0.44 for firing area, 0.33 for patchiness, and -0.08 for coherence. The correlation
Table 4. Comparisons of mean optimizing shifts

\begin{tabular}{llll} 
Measure & $d f$ & $t^{a}$ & $P(t)$ \\
\hline Firing area & 88 & 3.29 & 0.00074 \\
Patchiness & 88 & 0.79 & 0.18 \\
Coherence & 88 & 0.91 & 0.22
\end{tabular}

${ }^{a}$ The $t$ values are based on the null hypothesis that the mean optimizing shifts for $\mathrm{CA} 1$ and $\mathrm{CA} 3 / 4$ cells are equal. The positive values of $t$ indicate that the mean shifts are greater for $\mathrm{CAl}$ cells.

coefficients between the mean optimizing shifts for the 10 cell pairs recorded during single sessions were found to be 0.61 for firing area, 0.82 for patchiness, and 0.15 for coherence. Thus, there is some indication that optimizing shifts are characteristic for individual animals, and a much stronger suggestion that optimizing shifts are consistent during a session.

\section{Comparison of the optimal shift for CAI and CA3/4 place cells}

Of the 90 units in the sample, 41 were CA1 pyramidal cells and 49 were CA3/4 pyramidal cells. The average optimizing shift was positive for all 3 measures, for both $\mathrm{CA} 1$ and CA3/4 cells (Tables 3,4). Because the pyramidal cells in these 2 regions of the hippocampus are distinguishable anatomically and biochemically, it is of interest to see if they differ with respect to the average optimizing shift. The average optimizing shift for CA1 cells is greater than CA3/4 cells according to all 3 quality measures (Tables 3, 4). The differences are small and not statistically unlikely for patchiness and coherence, but the difference for firing area is $120 \mathrm{msec}[t=3.29 ; d f=88 ; P(t)=0.00074]$.

\section{Discussion}

\section{General considerations}

The central finding in this paper is that a positive spike/position shift of about $120 \mathrm{msec}$ is required to optimize the spatial firing patterns of hippocampal place cells. In other words, it is necessary to associate spikes with positions that occur $120 \mathrm{msec}$ later than the time of the spikes for the spatial firing pattern to appear most orderly. The implication is that spikes lead the animal's position by the stated interval; the spikes may be said to predict the animal's location, although the relationship is not necessarily causational.

Even if the temporal relationship between spikes and position were better established, it still could not be concluded that place cells are part of the causal chain that determines the animal's paths in its environment. From elementary considerations, a positive correlation between earlier neuronal activity and later motor output cannot prove that the one causes the other. The work of Berger and his colleagues (Berger et al., 1976, 1983; Berger and Thompson, 1978) on conditioning of the rabbit nictitating membrane response to an air puff provides a clear example of this principle. It was found that as conditioning proceeds, hippocampal pyramidal cells begin to fire such that the envelope of their activity after the conditional stimulus is remarkably similar to the time course of the conditioned movement; the neuronal activity forms a template of the motor response. Moreover, the neuronal activity precedes the response. Berger and Orr (1983) went on to show, however, that bilateral hippocampal ablations do not affect the conditioning despite the intimate relationship between cellular and motor activity. The fact that hippocampal lesions impair the ability of rats to 
solve spatial problems does, however, suggest that place cell firing is involved in the selection of trajectories (O'Keefe and Nadel, 1978; Morris et al., 1982; Sutherland et al., 1982).

Having acknowledged that motor-like causality cannot be proved from the positive shift, it is worth noting that the interval between spikes and location is long enough that place cell activity could conceivably have a role in controlling the animal's locomotor behavior. By this we mean that the minimal neural delay between the firing of hippocampal pyramidal cells and motor neurons may well be less than $120 \mathrm{msec}$. There are several known paths from the hippocampus to motor structures which could be fast enough to allow a direct hippocampal influence on motion. One involves the CA1 relay to the subiculum; the subiculum projects to retrosplenial cortex (Sorenson, 1980), which in turn projects to the ventral pontine nuclei (Domesick, 1969). Saint-Cyr and Woodward (1980) showed that stimulation of the fornix can activate Purkinje cells with a minimal latency of $5 \mathrm{msec}$ via the mossy fibers and a minimal latency of $10 \mathrm{msec}$ via the climbing fibers. Second, there is a pathway from the subiculum to nucleus accumbens (Swanson and Cowan, 1977), which in turn projects to the ventral pallidum (Heimer and Wilson, 1975). Finally, there are potential motor pathways via the entorhinal cortex, which projects to the striatum and the whole neocortex (Swanson and Këhler, 1986).

An alternative to the motoric interpretation is that place cells are very high order sensory neurons. In this view, it is imagined that place cells are triggered by complex stimulus configurations that occur all around the boundaries of the firing field. If this is true and if the animal walks forward, place cell firing could again be fastest before the animal reaches the field center, so that again a positive spike/position shift would improve field quality. The sensory and motor hypotheses can be distinguished by looking for trajectories that start with a movement directly towards the field center and that do or do not carry the rat through the center. The sensory interpretation suggests that the firing should be the same, irrespective of whether the rat goes through the field center. By contrast, the motor interpretation predicts that firing will be high only if the rat subsequently goes through the center. On this basis and others mentioned below, we conclude it is important to correlate ongoing place cell firing with specific trajectories; it is not enough to look only at the time-averaged activity.

A third interpretation of the positive spike/position shift is that the tracking headlights were not forward enough on the animal's head. Under this hypothesis, spikes lead position only because the chosen light position is not the same as the point on the rat's head used in calculations by place cells. Thus, the "correct" headlight placement might be at the rat's nose rather than between its ears. With some running speeds, a $2 \mathrm{~cm}$ forward movement of the headlights might change the required shift to zero or to a negative value. More interestingly, if running speed is taken into account, it could turn out that the "correct" location is a point in front of the animal's body. These considerations suggest that speed as well as path must be analyzed for a complete description of place cell firing, even if it turns out that the firing rate is independent of speed.

The arbitrariness of the point used to track the rat implies that spatial firing patterns should be optimized at a variety of light placements. The "proper" temporal shift would be where a combination of headlight placement and shift lead to the best spatial fring pattern. Assuming that the observed shift is due solely to "improper" headlight placement is a special case; it predicts that the optimum for the entire placement-shift plane should occur at the placement where the required shift is zero. We intend to examine this question by recording from place cells while tracking 2 lights horizontally placed $8 \mathrm{~cm}$ apart along the midline of the head. With 2-light data, it is possible to optimize the temporal shift at any point along the line between the 2 lights by interpolating or extrapolating.

\section{Variations of the optimal shift}

On average, the shift necessary to optimize spatial firing patterns was the same for all 3 field quality measures. Nevertheless, the shift varied greatly from cell to cell. There are 2 interpretations of the dispersion of the optimum. On the one hand, the variation may reflect noisiness in the data or insensitivity of the measures; the "true" optimal shift could be the same for all cells. On the other hand, the optimum might really vary among cells, suggesting that the place cell representation of the environment has temporal extent, a fascinating possibility. Note that cell specificity with regard to shift cannot be rejected because of the rather low positive correlations among the 3 field quality measures; the low correlations may mean that the measures are independent of each other, and not just that they are noisy.

$A$ related question concerns the differences between $\mathrm{CAl}$ and CA3/4 cells. The positive shift required for optimizing spatial firing patterns was greater for CA1 than for CA3/4 cells, although the difference between the mean shifts for cells in the 2 regions was considered reliable only for firing area. The result is interesting since $\mathrm{CA} 3 / 4$ pyramidal cells provide much of the synaptic input to CA1 pyramidal cells. On this basis alone, a smaller positive shift might have been expected for CAl cells.

\section{Asymmetry of the field quality versus shift function}

When field quality is plotted against shift over the range -1.0 to $+1.0 \mathrm{sec}$, it is found that field quality degrades in both directions away from the optimum, but that the rate of degradation is slower for shifts positive to the optimum. The effect is evident for firing area and is detectable for the other measures. The asymmetry is independent of the optimal shift and cannot result from incorrect headlight placement. The asymmetry reinforces the idea that place cells are better at predicting than they are at representing what has already happened. The origin of the asymmetry almost certainly lies in the temporal firing pattern exhibited by place cells as the animal moves across firing fields. Thus, the need to analyze firing during individual trajectories arises again.

\section{On the causality of place cell firing}

We have argued previously (Muller and Kubie, 1987) that place cells are not well described as either sensory or motor. In this view, place cells serve a higher-order, "cognitive" function, such that the firing properties reflect abstract information about the environment and the rat's position within the environment. It is therefore of interest to seek an interpretation of the optimal shift that does not depend on the sensorimotor dichotomy. In particular, we will give an interpretation that depends on the variations in optimal shift from cell to cell.

We propose that the activity of place cells is concerned with "what might be," as well as with "what is." We speculate, in other words, that the hippocampus calculates a set of possible solutions to the problem of getting from a starting position to a goal, such that cells with different optimal shifts are concerned with different solutions. If the hippocampus helps to select routes 
through the environment, it may be efficient to calculate many possible routes simultaneously, so that a change of the problem rules need not cause a long lag in finding a new solution. In this view, the currently selected route would be computed with the highest resolution (i.e., most cells). Alternatives to the current route would be computed with lower resolution (fewer cells), according to the extent to which they differ from the current route. It is interesting that these considerations also apply to the cerebellum, where the highly repetitive structure may allow the parallel computation of alternative motions, so that little time would be lost for recomputation when the position of a moving goal changes or an error is detected. This leap of faith has implications for the issue of how to allocate the resources of parallel computing machines.

\section{Extension of the shift method to other problems}

Depending on the neurophysiological problem, one of several methods can be used to estimate the delay between the activity of a single neuron and an earlier or later external state. For example, perievent spike histograms are constructed if a repetitive external event is available for time locking. Alternatively, if an external function of one variable changes in an uncontrolled way, its value can be cross-correlated with the spike train, and the latency can be measured from the time shift necessary to maximize or minimize the cross-correlation. Optimizing the cross-correlation was elegantly used by Houk et al. (1987) to estimate the latency between the firing of cells in the red nucleus and the EMG activity of arm muscles used by restrained cats in a food retrieval task. Urs Schwartz (personal communication) has used convolution methods to measure the degree of correlation between vestibular units and horizontal or vertical eye position and velocity, and also to measure the effective latency between firing and the state of the eye.

The method used here is a useful generalization of crosscorrelation for the case that the external state is a function of 2 (or more) variables, so that the firing correlate cannot be described with a single number. The method makes 2 assumptions:

1. There is well-defined model of how the firing is related to the external state. Here, it was assumed that firing rate as a function of the rat's position in the plane is smooth and that it monotonically decreases away from a single maximum, the field center.

2. There is at least one numerical measure of how closely the observed relationship conforms to the ideal. Here, 3 characteristics of ideal firing fields were used to estimate the relative extent to which firing patterns associated with different shifts approximated the ideal relationship.

It will be valuable to repeat this 2 -dimensional analysis with generalizations of the more formal methods used by Houk et al. (1987) and by Schwarz. Nevertheless, the notion of measuring a critical time by optimizing the association between neu- ronal firing and a multidimensional behavioral state is promising.

\section{References}

Berger, T. W., and J. L. Bassett (1989) Subcortical efferents of the posterior cingulate gyrus. J. Comp. Neurol. (in press).

Berger, T. W., and W. B. Orr (1983) Hippocampectomy selectively disrupts discrimination reversal conditioning of the rabbit nictitating membrane response. Behav. Brain Res. 8: 49-68.

Berger, T. W., and R. F. Thompson (1978) Identification of pyramidal cells as the critical elements in hippocampal neuronal plasticity during learning. Proc. Natl. Acad. Sci. USA 75: 1572-1576.

Berger, T. W., B. Alger, and R. F. Thompson (1976) Neuronal substrate of classical conditioning in the hippocampus. Science 192: 483485.

Berger, T. W., P. Rinaldi, D. J. Weisz, and R. F. Thompson (1983) Single unit analysis of different hippocampal cell types during classical conditioning of the rabbit nictitating membrane response. J. Neurophysiol. 50: 1197-1219.

Domesick, V. B. (1969) Projections from the cingulate cortex in the rat. Brain Res. 169: 286-320.

Heimer, L., and R. D. Wilson (1975) The subcortical projections of allocortex: Similarities in the neural associations of the hippocampus, the piriform cortex and the neocortex. In Proc. Golgi Cent. Symp., M. Santini, ed., pp. 177-193, Raven, New York.

Houk, J. C., D. A. Dessem, L. E. Miller, and E. H. Sybirska (1987) Correlation and spectral analysis of relations between single unit discharge and muscle activities. J. Neurosci. Methods 21: 201-224.

Kubie, J. L. (1984) A driveable bundle of microwires for collecting single-unit data from freely moving rats. Physiol. Behav. 32: 115118.

Morris, R. G. M., P. Garrud, J. N. P. Rawlins, and J. O'Keefe (1982) Place navigation impaired in rats with hippocampal lesions. Nature 297: $681-683$.

Muller, R. U., J. L. Kubie, and J. B. Ranck, Jr. (1987) Spatial firing patterns of hippocampal complex-spike cells in a fixed environment. J. Neurosci. 7: 1935-1950.

O'Keefe, J. (1979) A review of the hippocampal place cells. Prog. Neurobiol. 13: 78-109.

O’Keefe, J., and J. Dostrovsky (1971) The hippocampal as a spatial map. Preliminary evidence from unit activity in the freely moving rat. Brain Res. 34: 171-175.

O'Keefe, J., and L. Nadel (1978) The Hippocampus as a Cognitive Map, Clarendon Press, Oxford, U.K.

Paxinos, G., and G. Watson (1981) The Rat Brain in Stereotaxic Coordinates, Academic, New York.

Saint-Cyr, J. A., and D. J. Woodward (1980) Activation of mossy and climbing fiber pathways to the cerebellar cortex by stimulation of the fornix in the rat. Exp. Brain Res. 40: 1-12.

Sorenson, K. E. (1980) Ipsilateral projection from the subiculum to the retrosplenial cortex in the guinea pig. J. Comp. Neurol. 193:893911.

Sutherland, R. J., B. Kolb, and I. Q. Whishaw (1982) Spatial mapping: Definitive disruption by hippocampal or medial frontal damage in the rat. Neurosci. Lett. 31: 271-276.

Swanson, L. W., and W. M. Cowan (1977) An autoradiographic study of the efferent connections of the hippocampal formation in the rat. J. Comp. Neurol. 172: 49-84.

Swanson, L. W., and C. Këhler (1986) Anatomical evidence for direct projections from the entorhinal cortex to the entire cortical mantle in the rat. J. Neurosci. 6: 3010-3023. 